\title{
Surface and interface shear horizontal acoustic waves in piezoelectric superlattices
}

\author{
A. Bousfia, E. H. El Boudouti, ${ }^{\text {a) }}$ D. Bria, and A. Nougaoui \\ Laboratoire de Dynamique et d'Optique des Matériaux, Faculté des Sciences, Université Mohamed $1^{e r}$, \\ Oujda, Morocco \\ B. Djafari-Rouhani \\ Laboratoire de Dynamique et Structure des Matériaux Moléculaires, UPRESA 8024, CNRS, \\ UFR de Physique, Université de Lille I, F59655 Villeneuve d'Ascq Cedex, France \\ V. R. Velasco \\ Instituto de Ciencia de Materiales, CSIC, Cantoblanco, 28049 Madrid, Spain
}

(Received 21 September 1999; accepted for publication 3 February 2000)

\begin{abstract}
The propagation of acoustic waves of shear horizontal polarization in infinite and semi-infinite superlattices made of two piezoelectric media is studied within a Green's function method. Localized modes induced by a free surface of the superlattice or a superlattice/substrate interface are investigated theoretically. These modes appear as well-defined peaks of the total density of states inside the minigaps of the superlattice. The spatial localization of the different modes is studied by means of the local density of states. The surface of the superlattice and the superlattice/substrate interface are considered to be either metallized or nonmetallized. We show the possibility of the existence of interface modes, which are without analogue in the case of the interface between two homogeneous media (the so-called Maerfeld-Tournois modes). We also generalize to piezoelectric superlattices a rule about the existence and number of surface states, namely when one considers two semi-infinite superlattices together obtained by the cleavage of an infinite superlattice, one always has as many localized surface modes as minigaps, for any value of the wave vector $k_{\|}$ (parallel to the interfaces). Specific applications of these results are given for $\mathrm{CdS}-\mathrm{ZnO}$ superlattices with a free surface or in contact with a $\mathrm{BeO}$ substrate. (C) 2000 American Institute of Physics. [S0021-8979(00)08409-7]
\end{abstract}

\section{INTRODUCTION}

The study of acoustic vibrations in piezoelectric superlattices (SLs) has received increased attention in the last decade $^{1-12}$ due to the unusual physical properties observed in these heterostructures in comparison with bulk materials. ${ }^{13-16}$ The propagation of bulk and surface acoustic waves in homogeneous piezoelectric materials has found wide application, for instance, in the realization of transducers or in filtering. Electromechanical resonators are directly inserted into the circuits, the vibration being maintained by the electric field. Several years ago, the usefulness of piezoelectric superlattices (SLs) as transducers in the high frequency range was demonstrated, ${ }^{17}$ showing the possible application of this type of material to fabricate high-frequency acoustic resonance devices.

On the other hand, it has been shown theoretically that the ideal SL, which consists of an infinite repetition of two alternating layers, needs to be modified to take into account the media surrounding the SL, such as: vacuum, cap layer, substrate,,.. . The presence of such inhomogeneities within the perfect SL gives rise to localized modes inside the minigaps separating the bulk bands. ${ }^{1-7}$ One can quote also the works of Alshits et al. ${ }^{8-11}$ on the propagation of acoustic

a) Author to whom correspondence should be addressed; electronic mail: boudouti@sciences.univ-oujda.ac.ma waves in finite SLs made up of identical piezoelectric or piezomagnetic layers separated by infinitely thin cladding layers with metallic or superconducting properties, respectively. This allows an analysis of the reflection-transmission spectrum due to multiple reflection at the boundaries between layers. These investigations were performed using the transfer matrix, ${ }^{1,2,8-11}$ the Hamiltonian system formalism, ${ }^{7}$ and the Green's function method. ${ }^{3-6}$ The latter method is quite suitable for studying the spectral properties of the composite materials; in particular, it enables us to calculate the total and local density of states (DOSs) in which the localized modes associated with the different perturbations cited above appear as well-defined peaks. We have previously applied such a formalism to the case of purely shear horizontal elastic waves associated with a semi-infinite SL or to its interface with a substrate. ${ }^{18,19}$ For these waves involving only one direction of vibration, the Green's function has been calculated analytically and the DOS has been obtained as a function of the frequency $\omega$ and the wave vector $k_{\|}$ (parallel to the interfaces). However, the direct calculation becomes very cumbersome once the shear horizontal waves couple to the electric potential, although it remains analytical. ${ }^{20}$ This is encountered also in the case of pure elastic waves polarized in the sagittal plane..$^{21,22}$

In this article, we will discuss shear horizontal waves associated with the free surface of a semi-infinite piezoelec- 
tric SL or with its interface with a piezoelectric substrate. Let us mention that surface elastic waves in piezoelectric SLs were studied before, ${ }^{2,3}$ however, to our knowledge, the variation of the density of states associated with the free surface of piezoelectric SLs has not yet been studied. In particular, we generalize a result obtained previously ${ }^{18}$ in the case of nonpiezoelectric SLs. Namely, by considering together the two complementary SLs obtained by cleavage of an infinite SL along a plane parallel to the interfaces, one always has as many localized surface modes as minigaps for any value of the wave vector $k_{\|}$(parallel to the interfaces). On the other hand, we show that in contrast to the case of an interface between two piezoelectric homogeneous media where interface modes are rather rare to find, ${ }^{16}$ localized modes with high degree of localization may exist at the interface between a piezoelectric SL and a piezoelectric substrate.

After a brief presentation of the model and the method of calculation in Sec. II, we give in Sec. III some numerical results for a CdS/ZnO SL with a free surface or in contact with a $\mathrm{BeO}$ substrate. Section IV summarizes the principal results of this work.

\section{MODEL AND METHOD OF CALCULATION}

The interface SL substrate under consideration here is composed of a semi-infinite SL formed out of a semi-infinite repetition of two different stabs $(i=1,2)$ within the unit cell $n$, the SL being in contact with homogeneous substrate $i$ $=s$. The interface SL/vacuum can be obtained as a particular case of the SL/substrate system by replacing the substrate with a vacuum. All the interfaces are taken to be parallel to the $\left(x_{2}, x_{3}\right)$ plane of a reference orthonormal basis set. A space position along the $x_{1}$ axis (perpendicular to the interfaces) in medium $i$ belonging to the unit cell $n$ is indicated by $\left(n, i, x_{1}\right)$, where $-\left(d_{i} / 2\right) \leqslant x_{1} \leqslant\left(d_{i} / 2\right)$, while $x_{1} \geqslant 0$ in the substrate. The layers and the substrate are characterized by their elastic, dielectric, and piezoelectric constants and by their mass densities. The thicknesses $d_{1}$ and $d_{2}$ of the layers are assumed to be equal without loss of generality, the period of the SL being $D=d_{1}+d_{2}$.

The media forming the layers of the SL and the substrate are assumed to be of hexagonal symmetry belonging to the 6 $\mathrm{mm}$ class with their $c$ axis along the $x_{3}$ axis and the wave vector $k_{\|}$, parallel to the interfaces, is along $x_{2}$. In this particular geometry, the shear horizontal vibrations (parallel to $x_{3}$ ) are accompanied by an electric potential, while the sagittal vibrations [polarized in the $\left(x_{1}, x_{2}\right)$ plane] are decoupled from the latter. ${ }^{1,2}$ Let us mention that surface and interface elastic waves of sagittal polarization in semi-infinite SLs were studied recently. ${ }^{21,22}$

We obtain the local and total DOS associated with the SL substrate interface from knowledge of the corresponding Green's function, which is calculated here by using the theory of interface response in composite materials. ${ }^{23}$ In this theory, the Green function $\mathbf{g}$ of a composite system can be written as ${ }^{23}$

$$
\begin{aligned}
\mathbf{g}(D D)= & \mathbf{G}(D D)+\mathbf{G}(D M)\left[\mathbf{G}^{-1}(M M) \mathbf{g}(M M) \mathbf{G}^{-1}(M M)\right. \\
& \left.-\mathbf{G}^{-1}(M M)\right] \mathbf{G}(M D),
\end{aligned}
$$

where $D$ and $M$ are, respectively, the whole space and the space of the interfaces in the composite material. $G$ is a block-diagonal matrix in which each block $\mathbf{G}_{i}$ corresponds to the bulk Green function of the subsystem $i$. In our case, the composite material is composed of a SL built out of alternating slabs of materials $i(i=1,2)$ with thickness $d_{i}$, in contact with a substrate of material $i=s$. In Eq. (1) the calculation of $\mathbf{g}(D D)$ requires, besides $\mathbf{G}_{i}$, knowledge of $\mathbf{g}(M M)$. In practice, the latter is obtained ${ }^{23}$ by inverting the matrix $\mathrm{g}^{-1}(M M)$, which can be simply built from a juxtaposition of the matrices $g_{s i}^{-1}(M M)$, where $g_{s i}^{-1}(M M)$ is the interface Green's function of the slabs $i(i=1,2)$ and of the substrate alone. ${ }^{23}$ The details of the analysis are the same as for shear horizontal waves in elastic superlattices, ${ }^{18,19}$ but with more complicated calculation. ${ }^{20}$ Let us emphasize that, in the geometry of the SL substrate structure, the elements of the Green's function take the form $g_{\alpha \beta}\left(\omega^{2}, k_{\|} \mid n, i, x_{1} ; n^{\prime}, i^{\prime}, x_{1}^{\prime}\right)$, where $\omega$ is the frequency of the acoustic wave, $k_{\|}$the wave vector parallel to the interfaces, and $\alpha, \beta$ denote the components of a $4 \times 4$ matrix $(\alpha, \beta=1,2,3,4)$ representing the coupling between the acoustic waves $(\alpha, \beta=1,2,3)$ and the electric field $(\alpha, \beta=4)$. For the sake of simplicity, we shall omit in the following the parameters $\omega^{2}$ and $k_{\|}$, and we note as $g\left(n, i, x_{1} ; n^{\prime}, i^{\prime}, x_{1}^{\prime}\right)$ the $4 \times 4$ matrix whose elements are $g_{\alpha \beta}\left(n, i, x_{1} ; n^{\prime}, i^{\prime}, x_{1}^{\prime}\right)(\alpha, \beta=1,2,3,4)$.

By assuming that $k_{\|}$is along the $x_{2}$ direction, the components $g_{\alpha \beta}(\alpha, \beta=1,2)$ of the Green function decouple ${ }^{20}$ from the components $g_{\alpha \beta}(\alpha, \beta=3,4)$ (i.e., $g_{13}=g_{14}=g_{23}$ $=g_{24}=g_{31}=g_{32}=g_{41}=g_{42}=0$;) the former corresponds to vibrations polarized in the sagittal plane, whereas the latter are associated with shear horizontal vibrations accompanied by an electric potential.

For the shear horizontal vibrations coupled to the electric potential studied here, knowledge of the Green's function $\mathbf{g}$ in the SL substrate system enables us to calculate the local DOS, for a given value of the wave vector $k_{\|}$

$$
\begin{aligned}
n_{\alpha}\left(\omega^{2}, k_{\|} \mid n, i, x_{1}\right)= & -\frac{1}{\pi} \operatorname{Im} g_{\alpha \alpha}\left(\omega^{2}, k_{\|} \mid n, i, x_{1} ; n, i, x_{1}\right) \\
& (\alpha=3,4),
\end{aligned}
$$

or

$$
\begin{aligned}
n_{\alpha}\left(\omega, k_{\|} \mid n, i, x_{1}\right)= & -\frac{2 \omega}{\pi} \operatorname{Im} g_{\alpha \alpha}\left(\omega^{2}, k_{\|} \mid n, i, x_{1} ; n, i, x_{1}\right) \\
& (\alpha=3,4) .
\end{aligned}
$$

The total DOS can be obtained either by integrating directly over the space variable $x_{1}$ or by using a method that involves only the interface matrix elements of the Green's functions. In the latter method, one can calculate the difference between the total DOSs of two complementary semiinfinite superlattices (terminating with layers $i=1$ and $i=2$ ) in contact with two substrates and the reference system (i.e., an infinite SL, and an infinite substrate), if we only know ${ }^{23}$ the elements of the Green's at the surface $M_{s}$ of the two complementary semi-infinite SLs and of the two substrates. By calling $d_{1}\left(M_{s}, M_{s}\right), d_{2}\left(M_{s}, M_{s}\right)$, and $g_{s}\left(M_{s}, M_{s}\right)$ these Green's functions matrix elements, one can write 


$$
\Delta n(\omega)=-\frac{1}{\pi} \operatorname{Im} \frac{d}{d \omega} \ln \operatorname{det}\left[\frac{\left[\mathbf{d}_{1}\left(M_{s}, M_{s}\right)+\mathbf{g}_{s}\left(M_{s}, M_{s}\right)\right]\left[\mathbf{d}_{2}\left(M_{s}, M_{s}\right)+\mathbf{g}_{s}\left(M_{s}, M_{s}\right)\right]}{2\left[\mathbf{d}_{1}\left(M_{s}, M_{s}\right)+\mathbf{d}_{2}\left(M_{s}, M_{s}\right)\right] \mathbf{g}_{s}\left(M_{s}, M_{s}\right)}\right] .
$$

As mentioned above, the interface SL/vacuum is obtained from Eq. (4) by replacing the Green's function $g_{s}$ of the substrate by the Green's function $g_{v}$ of vacuum. We shall distinguish also, as in usual piezoelectric materials, ${ }^{14-16}$ two types of surface boundary conditions depending on whether the surface is metallized or not.

\section{DISCUSSION OF THE RESULTS}

In the following, we shall give some specific illustrations of these results. In these examples, the SL is made of CdS and $\mathrm{ZnO}$ with a free surface or in contact with a $\mathrm{BeO}$ substrate, having a high transverse velocity. The characteristic parameters of the materials used here are listed in Table I. For given $\omega$ and $k_{\|}$, the wave vectors along the axis $x_{1}$ of the SL, which can be deduced from the bulk dispersion, are called $k_{1}$. In the case of piezoelectric waves involving two components of the displacement vector and the electric potential, there are two pairs of values of $k_{1}$ with given $k_{\|}$and $\omega$, which can be written as ${ }^{1,2} \pm\left(K_{1}^{\prime}+i L_{1}^{\prime}\right)$ and $\pm\left(K_{2}^{\prime}\right.$ $\left.+i L_{2}^{\prime}\right)$. Now an elastic wave with a frequency $\omega$ will propagate in the SL if $L_{1}^{\prime}=0$ or $L_{2}^{\prime}=0$, while it is attenuated if both $L_{1}^{\prime}$ and $L_{2}^{\prime}$ are different from zero. Each pair of values of $k_{1}$ (the first for instance) can take four different forms

(i) pure real $\left(L_{1}^{\prime}=0\right)$,

(ii) pure imaginary $\left(K_{1}^{\prime}=0\right)$,

(iii) complex but with $K_{1}^{\prime}= \pm \pi / D$,

(iv) complex with $K_{1}^{\prime} \neq \pm \pi / D$.

However, in case (iv) the two pairs of $k_{1}$ necessarily become

$$
K^{\prime}+i L^{\prime},-\left(K^{\prime}+i L^{\prime}\right) ; K^{\prime}-i L^{\prime},-\left(K^{\prime}-i L^{\prime}\right) \text {. }
$$

Figures 1(a) and 1(b) give two examples of the complex band structure (thin solid curves) in a CdS/ZnO SL showing combinations of the above mentioned cases for small and large values of $k_{\|} D$, namely, $k_{\|} D=0.2$ [Fig. 1(a)] and $k_{\|} D$ $=4$ [Fig. 1(b)]. One can see the dispersion of the displacement component in Figs. 1(a) and 1(b) giving rise to direct gaps at the center and at the edge of the reduced Brillouin zone, while the potential component is dispersionless and appears as a vertical line at the imaginary part of $k_{1}$ such that $\operatorname{Im}\left(k_{1} D\right)=k_{\|} D$. However, for small values of $k_{\|} D$, the potential curve may interact with the vibrational one leading to the lifting of the degeneracy at the crossing points as is the case

TABLE I. Elastic, piezoelectric and dielectric parameters of the materials $\mathrm{CdS}, \mathrm{ZnO}$, and $\mathrm{BeO}$.

\begin{tabular}{ccccc}
\hline \hline Material & $\begin{array}{c}C_{44} \\
\left(10^{10} \mathrm{~N} / \mathrm{m}^{2}\right)\end{array}$ & $\begin{array}{c}\rho \\
\left(\mathrm{kg} / \mathrm{m}^{3}\right)\end{array}$ & $\begin{array}{c}e_{15} \\
(\mathrm{C} / \mathrm{m})\end{array}$ & $\begin{array}{c}\epsilon_{11} \\
\left(10^{-11} \mathrm{~F} / \mathrm{m}\right)\end{array}$ \\
\hline $\mathrm{Cds}$ & 1.49 & 4824 & -0.21 & 7.99 \\
$\mathrm{ZnO}$ & 4.25 & 5676 & -0.59 & 7.38 \\
$\mathrm{BeO}$ & 14.77 & 3009 & - & 5.99 \\
\hline \hline
\end{tabular}

in the second gap of Fig. 1(a). As a matter of comparison, we have also plotted in Fig. 1(a) the complex band structure associated with pure transverse elastic waves (heavy solid curves), i.e., when the piezoelectric constant $e_{15}$ is taken equal to zero. The dispersion curves are slightly shifted toward lower frequencies at small values of $k_{\|} D$ [Fig. 1(a)]; however, the shifting disappears for large values of $k_{\|} D$ [Fig. 1(b)], where we have not plotted the curves associated with the pure elastic waves.

Now, the creation of the free surface of the SL gives rise to localized modes inside the minigaps of the SL; their frequencies, indicated by arrows in Figs. 1(a) and 1(b), are very dependent upon the composition of the layer that terminates the SL. The full (dashed) arrows in Fig. 1 represent the surface modes when a $\mathrm{CdS}(\mathrm{ZnO})$ film is at the surface of the $\mathrm{SL}$; moreover if the surface is metallized, the surface modes
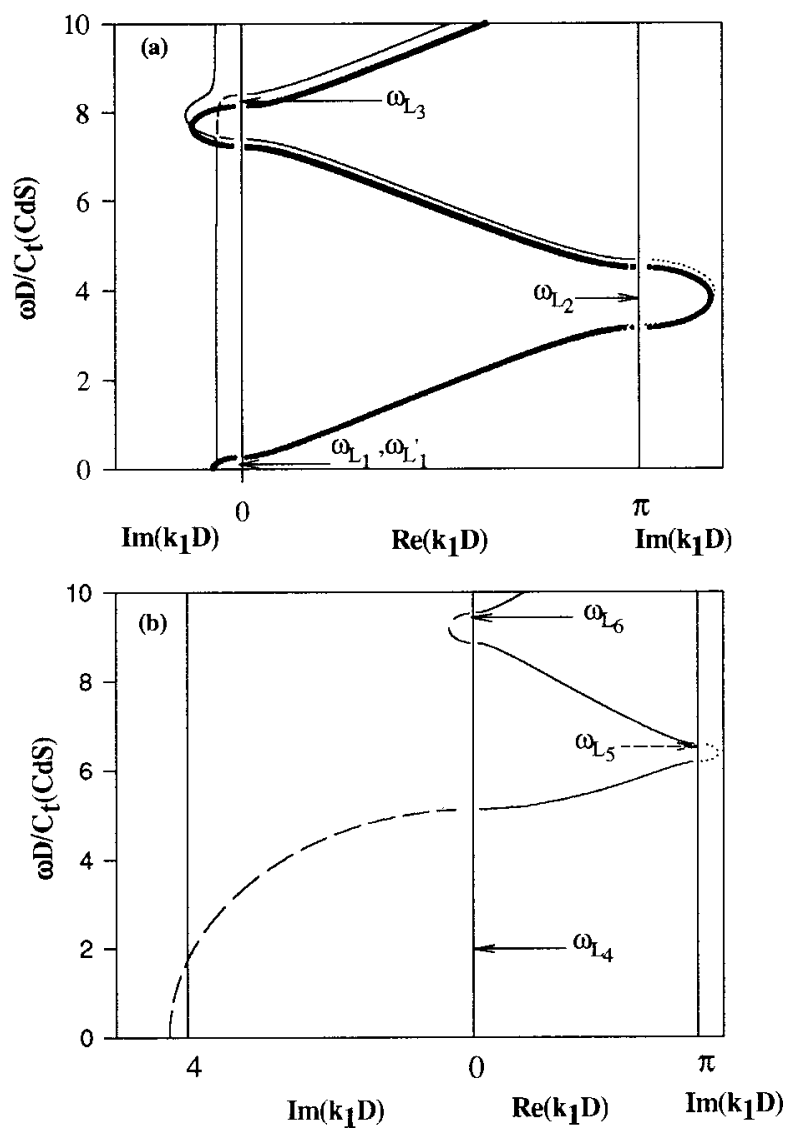

FIG. 1. Complex band structure ( $\omega$ vs complex $k_{1}$ ) in a CdS/ZnO SL with $d_{1}=d_{2}=D / 2$ and $k_{\|} D=0.2(\mathrm{a}), 4(\mathrm{~b}), \omega D / C_{t}(\mathrm{CdS})$ is a dimensionless frequency where $C_{t}(\mathrm{CdS})$ is the transverse velocity of sound in CdS given by $\left(C_{44} / \rho\right)^{1 / 2}$. Thin solid curves are $k_{1}$ real (middle panel of the figure). Dashed curves are $k_{1}$ imaginary (left panel). Dotted curves are the imaginary part of $k_{1}$ when its real part is equal to $\pi / D$ (right panel). Heavy solid curves in (a) represent the complex band structure but neglecting the piezoelectricity. The horizontally full (dashed) arrows give the frequencies of the surface modes inside the minigaps of the SL when the layer at the surface of the $\mathrm{SL}$ is $\mathrm{CdS}(\mathrm{ZnO})$. 


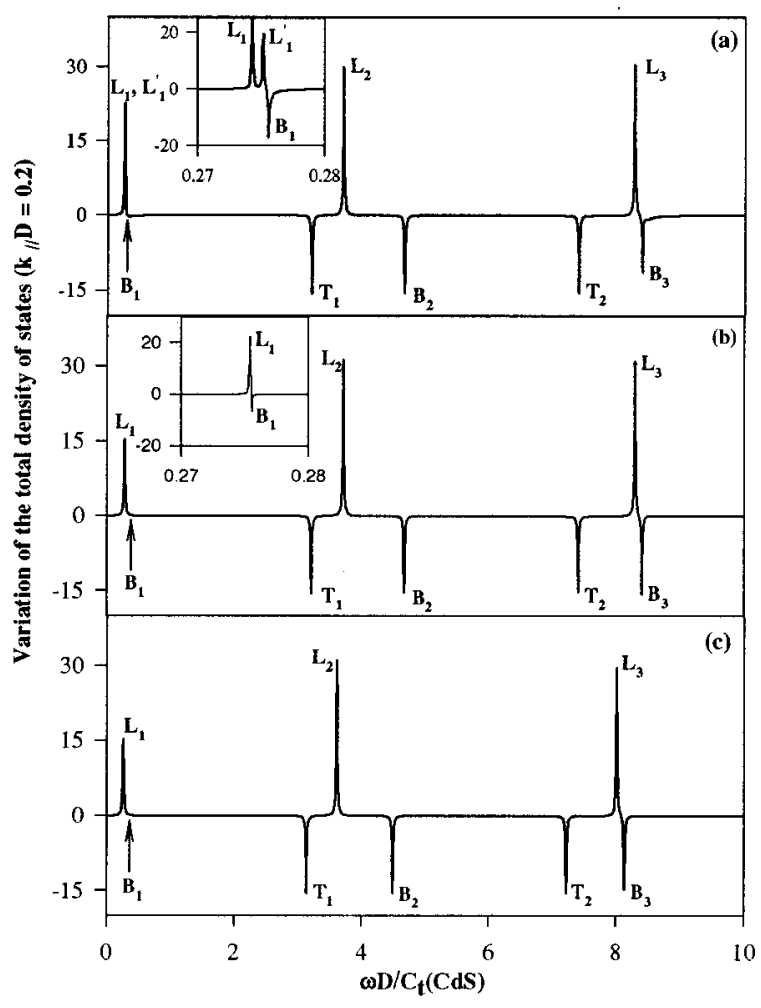

FIG. 2. Variation of the DOS [in units of $D / C_{t}(\mathrm{CdS})$ ] when creating two complementary semi-infinite SLs from the infinite SL, for $k_{\|} D=0.2$. Panels (a), (b), and (c) correspond, respectively, to the case when the films at the surfaces of the SLs are metallized, nonmetallized, and pure elastic. $L_{1}, L_{2}$, and $L_{3}$ represent surface modes when CdS is at the surface of the SL. $L_{1}$ corresponds to the surface mode when $\mathrm{ZnO}$ is at the surface; it appears when the surface is metallized. $B_{i}$ and $T_{i}$ correspond to $\delta$ peaks of weight $-1 / 2$ which appear, respectively, at the bottom and the top of each bulk band. In the low-frequency range, the surface modes are located very close to the limits of the bulk bands and therefore mask the band-edge antiresonances. Therefore, the low-frequency region is enlarged in the inserts of panels (a) and (b).

change slightly but cannot be distinguished from the preceding ones at the scale of the figure. Let us mention that in the small range of $k_{\|} D$ [Fig. 1(a)], there are two surface modes (labeled $\omega_{L_{1}}$ and $\omega_{L_{1}}^{\prime}$ ) lying below the bulk bands. These modes may exist for both $\mathrm{CdS}$ and $\mathrm{ZnO}$ terminations of the SL depending on whether the surface is metallized or not (see details below).

The surface modes are poles of the Green function and therefore appear as $\delta$ peaks in the DOS as illustrated in Fig. 2. In this figure, we have presented, for $k_{\|} D=0.2$, the variation of the total DOS when two complementary semi-infinite SLs are created by the cleavage of an infinite SL in such a way that one obtains one SL with a full $\mathrm{CdS}$ layer at the surface and its complementary one with a full $\mathrm{ZnO}$ layer at the surface. For the sake of clarity and despite the analytic nature of our calculation, the $\delta$ peaks in the DOS are broadened by adding a small imaginary part to the frequency $\omega(\omega \rightarrow \omega+i \epsilon)$. Figures 2(a)-2(c), are respectively, associated with the cases where the surface of the SL is metallized [Fig. 2(a)], nonmetallized [Fig. 2(b)], and pure elastic [Fig. 2(c)]. $L_{i}$ are $\delta$ peaks associated with surface localized modes, whereas $B_{i}$ and $T_{i}$ refer to $\delta$ peaks of weight $(-1 / 2)$ situated at the bottom and the top of the minibands ${ }^{18-22}$ given by

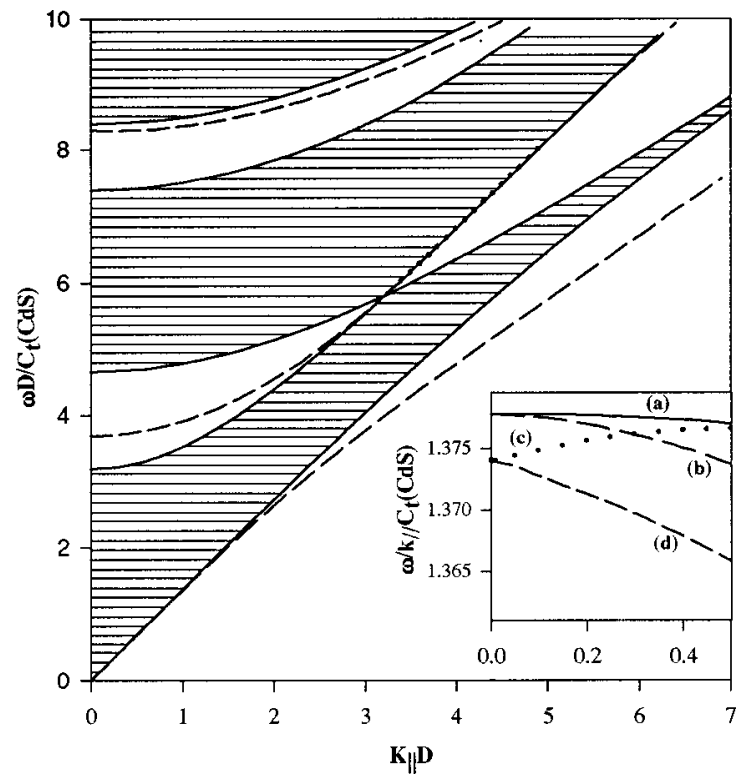

FIG. 3. Bulk band and surface wave dispersion for a $\mathrm{CdS} / \mathrm{ZnO}$ SL with $d_{1}=d_{2}$. The curves give $\omega D / C_{t}(\mathrm{CdS})$ as a function of $k_{\|} D$, where $\omega$ is the frequency, $k_{\|}$the propagation vector parallel to the interfaces. The shaded areas are the bulk bands. The dashed (dotted) curves represent the surface modes when a nonmetallized $\mathrm{CdS}(\mathrm{ZnO})$ layer is at the surface. For a metallized surface, the surface modes are slightly different but cannot be distinguished from the preceding ones at the scale of the figure. The insert shows, in units of $C_{t}(\mathrm{CdS})$, the phase velocities of the bottom of the bulk bands (a) and of the surface waves below it [(b), (c) and (d)] over a small range of $k_{\|} D$. The curves (b), (d) and (c), respectively, correspond to the case when the film at the surface is CdS nonmetallized, CdS metallized, and $\mathrm{ZnO}$ metallized.

$k_{1} D=0$ and $k_{1} D=\pi$. One can see that the positions of the surface modes are almost the same in the first two cases. However, there exists two (respectively, one) surface modes below the bulk bands in the case of metallized (respectively, nonmetallized) surfaces [see the inserts in Figs. 2(a) and 2(b)]; these modes, labeled $L_{1}$ and $L_{1}^{\prime}$, are associated with $\mathrm{CdS}$ and $\mathrm{ZnO}$ terminations of the SL, respectively. Figure 2(c), corresponding to the pure elastic case, shows a shift of the bulk bands and surface modes toward lower frequencies.

In Fig. 3 we represent the so-called projected band structure of the bulk bands and surface modes, namely, $\omega$ versus $k_{\|}$. The shaded areas represent bulk bands separated by minigaps. Inside these gaps, we have represented surface modes corresponding to the two complementary semiinfinite SLs with CdS termination (dashed curves) and $\mathrm{ZnO}$ termination (dotted curves). As mentioned above, the frequencies of the surface modes are almost the same in the case of metallized and nonmetallized surfaces. A particular situation occurs in the long wavelength limit (see the inset of Fig. 3): when the SL terminates with a CdS layer, the surface mode below the bulk band exists whether the surface is metallized or nonmetallized; on the other hand, for a $\mathrm{ZnO}$ termination of the SL, the surface mode exists over a short range of the dimensionless wave vector $k_{\|} D$ only if the surface is metallized. These results are in accordance with those presented in the insets of Figs. 2(a) and 2(b). From the results given in Fig. 3, one should notice that apart from the long wavelength region, there are as many surface states as 

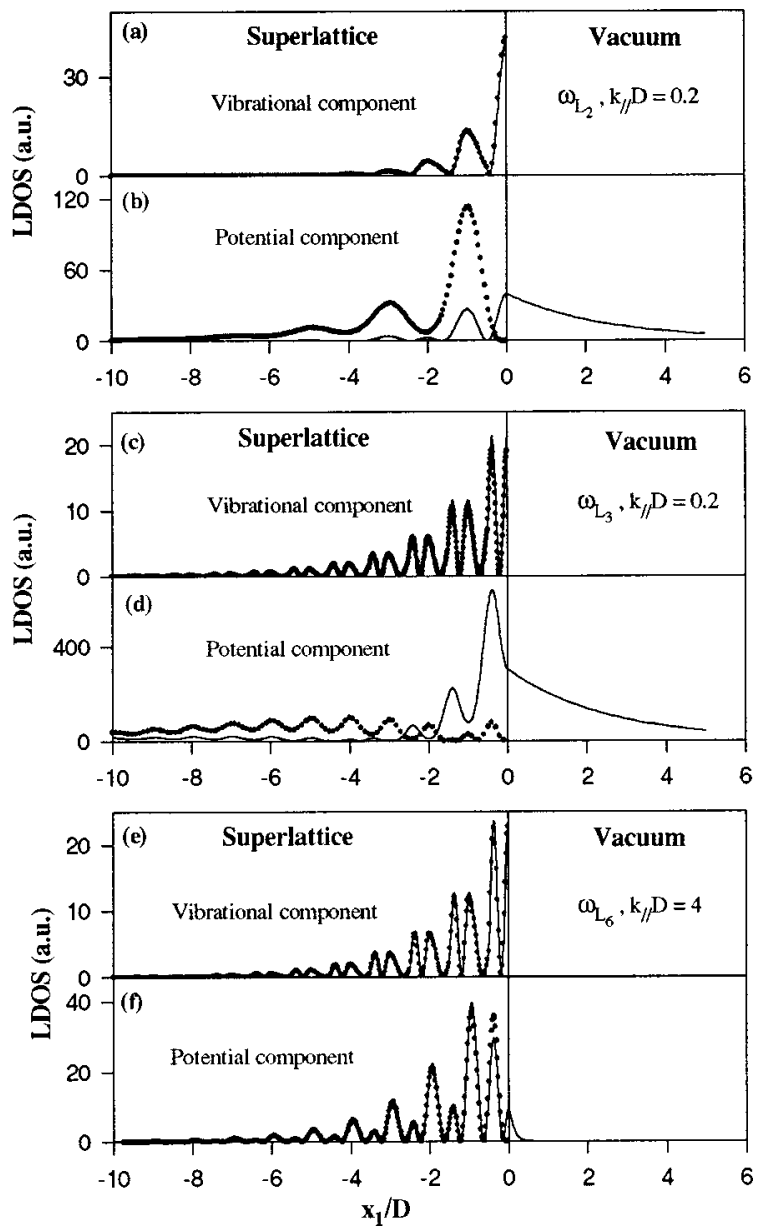

FIG. 4. Spatial representation of the local DOS for the modes labeled $\omega_{L 2}$ [(a), (b)], $\omega_{L 3}[(\mathrm{c}),(\mathrm{d})]$, and $\omega_{L 6}$ [(e), (f)] in Fig. 1. The curves in (a), (c), and (e) [respectively (b), (d), and (f)] correspond to the vibrational (respectively potential) components of the local DOS. The full (dotted) curves correspond to the case when the film at the surface is CdS metallized (CdS nonmetallized)

minigaps for each value of the wave vector $k_{\|}$. Each surface mode is associated with either one or the other of the complementary semi-infinite SLs. This result is obtained numerically based on the following two steps: (i) the variation of the DOS when creating two complementary semi-infinite SLs from an infinite one is equal to zero for any frequency $\omega$ falling inside a SL bulk band (ii) there is a loss of $1 / 2$ state at every edge of a bulk band (see Fig. 2), i.e., a loss of one state per miniband. Therefore, to ensure the conservation of the total number of states, it is necessary to have as many localized modes as minigaps in the band structure. The above rule generalizes a similar result obtained previously by some of the authors ${ }^{18}$ for nonpiezoelectric SLs.

To illustrate the spatial localization of the surface modes, we have sketched in Fig. 4 the local DOS associated with the modes labeled $\omega_{L 2}, \omega_{L 3}$, and $\omega_{L 6}$ in Fig. 1: the DOS is presented as a function of the space position $x_{1}$, at $k_{\|} D=0.2$ and $k_{\|} D=4$. Both the vibrational and the electric components of the DOS are given for two types of electric boundary conditions, namely a metallized and a nonmetallized surface. The displacement field is not greatly affected by the electric boundary condition, while the electric field

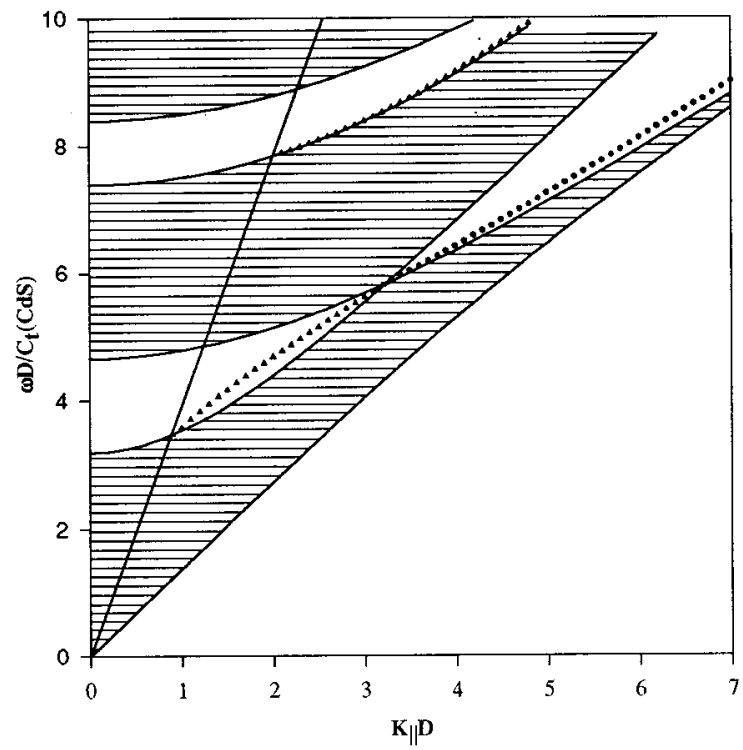

FIG. 5. Interface localized modes associated with a CdS/ZnO SL in contact with a $\mathrm{BeO}$ substrate. The shaded areas are the bulk bands of the SL. The heavy straight line indicates the bottom of the substrate bulk band. When the $\mathrm{SL}$ terminates with a $\mathrm{ZnO}$ (respectively $\mathrm{CdS}$ ) layer, the localized modes are represented by the triangles (dotted curves).

shows different behaviors for a metallized or a nonmetallized surface. However, these differences become less important when going to higher values of $k_{\|} D$. For both vibrational and electric components of the surface wave, one should notice an exponential decrease of their envelopes when penetrating deep into the interior of the SL; this attenuation is accompanied by an increasing number of oscillations in each period of the SL as far as one is dealing with higher frequency modes.

In the following we show for the first time to our knowledge, the possibility of the existence of piezoelectric waves localized at the interface between a piezoelectric SL and a piezoelectric substrate.

The results will be illustrated for the same CdS/ZnO SL in contact with a $\mathrm{BeO}$ substrate. The velocity of the transverse speed of sound in the $\mathrm{BeO}$ substrate is rather high, which enables us to give a qualitative description of the different localized modes induced by the SL/substrate interface, inside the minigaps and below the substrate bulk band.

Figure 5 gives the interface modes for both the complementary SLs in which the substrate is either in contact with a $\mathrm{ZnO}$ layer (triangles) or with a CdS layer (dotted curves). One can clearly notice that the frequencies of the interface modes are very sensitive to the type of layer which is at the surface of the SL. Let us mention that, to our knowledge, the piezoelectric constant $e_{15}$ of the $\mathrm{BeO}$ substrate is not available in the literature. ${ }^{13}$ However, we have determined that physically acceptable values of the substrate piezoelectric constant $e_{15}$ (Ref. 13) do not affect the frequencies of the interface modes.

As in the case of a free surface, one can distinguish two types of boundary conditions (metallized or nonmetallized interface). The interface modes in Fig. 5 are very close for both boundary conditions and cannot be distinguished at the 


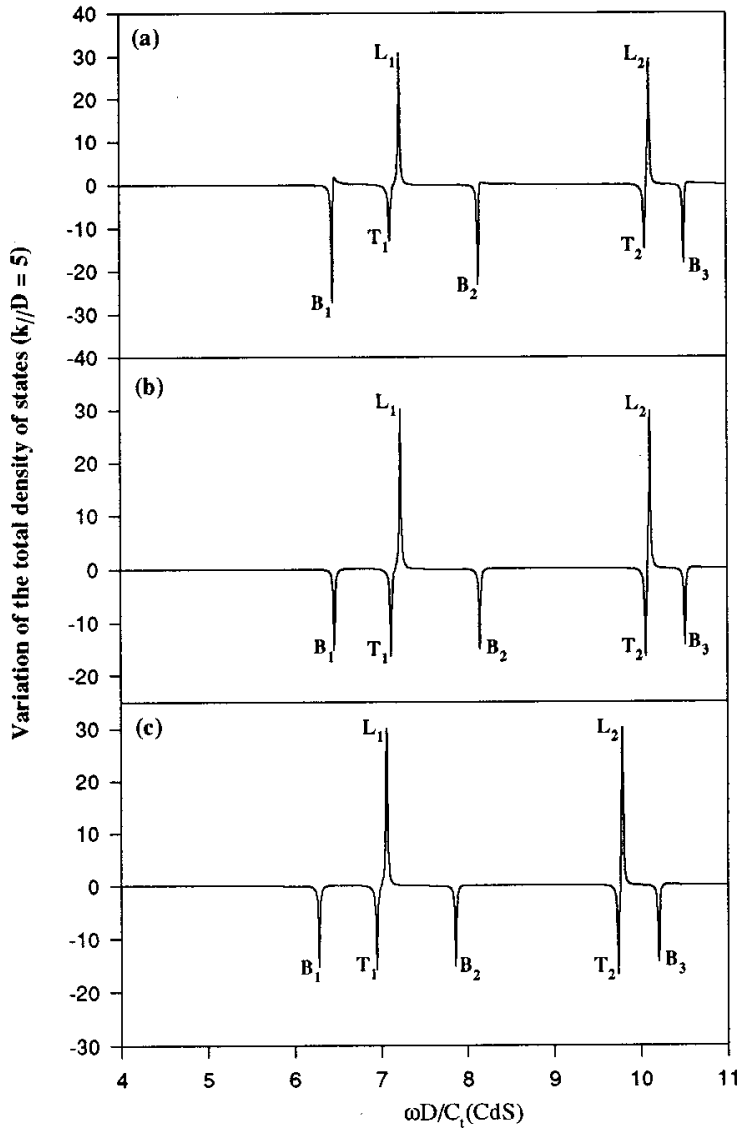

FIG. 6. Variation of the DOS at $k_{\|} D=5$, for the two complementary SLs of Fig. 5 created from the infinite SL and the infinite substrate. $L_{1}$ and $L_{2}$ represent interface modes associated with $\mathrm{CdS}$ and $\mathrm{ZnO}$ terminations of the SL respectively, whereas $B_{i}$ and $T_{i}$ have the same meaning as in Fig. 2.

scale of Fig. 5. However, the frequencies of the interface modes slightly shift toward lower frequencies when the piezoelectric coupling is not taken into account as it can be seen in Fig. 6. In this figure we have plotted, at $k_{\|} D=5$, the variation of the total DOS for the metallized [Fig. 6(a)] nonmetallized [Fig. 6(b)], and pure elastic [Fig. 6(c)] cases. The latter case is obtained by taking the piezoelectric constants equal to zero in the media constituting the SL and the substrate. The interface modes labeled $L_{1}$ and $L_{2}$ in Fig. 6 are associated with $\mathrm{CdS}$ and $\mathrm{ZnO}$ terminations of the SL, respectively.

The spatial localization of the interface mode $L_{1}$ is illustrated in Fig. 7 where we have presented both the vibrational and electric components of the DOS. The vibrational component is not affected by the electric boundary condition, while the behavior of the electric component is slightly different in the vicinity of the interface for both types of boundary conditions. Let us mention finally that the interface modes between two homogeneous piezoelectric media (the so-called Maerfeld-Tournois modes) are rather rare to find. ${ }^{16}$ In our case, for instance, the interface $\mathrm{BeO} / \mathrm{CdS}$ and $\mathrm{BeO} /$ $\mathrm{ZnO}$ do not support interface modes. Therefore, the SL/ substrate interface presents a useful structure for the existence of interface modes in comparison with the interface between two homogeneous media.

\section{SUMMARY}

In summary, the main results of this work, apart from the analytical derivation of the Green's function and the corresponding $\operatorname{DOS}^{20}$ are:

(i) The generalization to piezoelectric SLs of a previous theorem about the existence and number of surface states. More precisely, we have shown that, apart from the longwavelength limit, one obtains as many localized surface states as minigaps for any value of the wave vector $k_{\|}$. This result is based on the general rule about the conservation of the total number of states and expresses a compensation between the losses of $1 / 2$ state at every bulk band edge (due to the creation of the free surfaces) and the gain due to the occurrence of surface states. The imaginary part of the $k_{1}$ wave vectors obtained in the complex band structure calculation, gives the attenuation of the surface waves in a semiinfinite SL. Although the frequencies of the localized modes associated with metallized and nonmetallized surfaces are almost the same, their spatial localization may exhibit noticeable differences, in particular, for small values of $k_{\|} D$.

(ii) The derivation of the dispersion curves and the localization properties of the interface modes at the SL/ substrate boundary, with specific application to the $\mathrm{CdS} / \mathrm{ZnO}$ piezoelectric SL in contact with a $\mathrm{BeO}$ piezoelectric substrate. The dispersion curves of the interface modes are very dependent upon the nature of the layer in the SL which is in contact with the substrate.

(iii) The interface between a homogeneous and a periodic layered media appears as a fruitful structure for the existence of interface modes in comparison with the interface between two homogeneous media where the existence of localized modes (called Maerfeld-Tournois modes) becomes rather hard to find. ${ }^{16}$ This opens, at least in principle, some
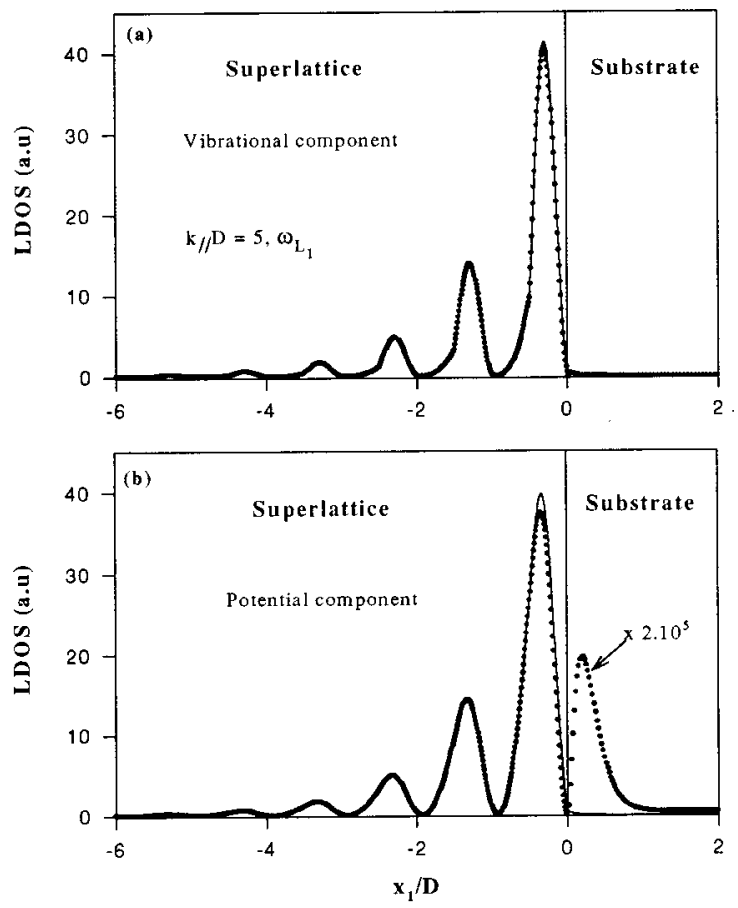

FIG. 7. Same as in Fig. 4, but for the interface mode labeled $L_{1}$ in Fig. 6. 
new opportunities for guided waves and filtering which is of potential value in the interface characterization of the multilayer/homogeneous structure.

Let us mention finally that the Green's function approach used in this analysis also enables us to obtain the reflection coefficients and the corresponding phase times associated with a reflected wave from an incident phonon in the substrate, even though we have not emphasized this aspect here. The experimental observation of the surface and interface modes predicted here can be performed by means of Brillouin scattering experiments ${ }^{24-26}$ and picosecond laser technique. ${ }^{27}$ Because many new devices are based on layered structures, ${ }^{28,29}$ it is important to understand and characterize propagation of waves in this kind of system. Therefore this work may be of interest in the field of the dynamics of piezoelectric layered structures and in particular for those applications which deal with the propagation of surface and interface acoustic waves in the high frequency range.

\section{ACKNOWLEDGMENTS}

The work of A.B., E.E., D.B., and A.N. has been supported by the Program-in-Aid for Scientific Research (PARS). The work of V.R.V. has been supported by the Dirección General de Enseñanza Superior (Spain) through Grant No. PB96-0916. E.E., A.N. and V.R.V. thank the CNCPRST (Morocco) and CSIC (Spain) for a cooperation project.

${ }^{1}$ J. Sapriel and B. Djafari-Rouhani, Surf. Sci. Rep. 10, 189 (1989).

${ }^{2}$ A. Nougaoui and B. Djafari-Rouhani, Surf. Sci. 185, 154 (1987).

${ }^{3}$ L. Fernández, V. R. Velasco, and F. García-Moliner, Surf. Sci. 188, 140 (1987).

${ }^{4}$ L. Fernández and V. R. Velasco, Surf. Sci. 185, 175 (1987).

${ }^{5}$ L. Fernández, V. R. Velasco, and F. García-Moliner, Europhys. Lett. 3, 723 (1987).
${ }^{6}$ J. E. Zárate, L. Fernández-Alvarez, and V. R. Velasco, Superlattices Microstruct. 25, 519 (1999).

${ }^{7}$ A. N. Podlipenets and L. P. Zinchuk, Funct. Mater. 2, 179 (1995).

${ }^{8}$ V. A. Alshits and A. L. Shuvalov, JETP 76, 663 (1993).

${ }^{9}$ V. A. Alshits and A. L. Shuvalov, Phys. Lett. A 177, 253 (1993).

${ }^{10}$ V. A. Alshits and A. L. Shuvalov, J. Appl. Phys. 177, 2659 (1995).

${ }^{11}$ V. A. Alshits, A. S. Gorkunova, and A. L. Shuvalov, JETP 83, 509 (1996).

${ }^{12}$ A. L. Shuvalov and A. S. Gorkunova, Phys. Rev. B 59, 9070 (1999).

${ }^{13}$ B. A. Auld, Acoustic Fields and Waves in Solids (Wiley, New York, 1973); Vol. I, p. 265. E. Dieulesaint and D. Royer, Ondes Elastiques dans les Solides (Masson, Paris, 1974); Handbook of Surfaces and Interfaces, edited by L. Dobrzynski (Garland STPM, New York, 1978), Vol. 2, p. 66.

${ }^{14}$ J. L. Bleustein, Appl. Phys. Lett. 13, 412 (1968).

${ }^{15}$ Yu. V. Gulyaev, JETP Lett. 9, 37 (1969).

${ }^{16}$ C. Maerfeld and P. Tournois, Appl. Phys. Lett. 19, 117 (1971).

${ }^{17}$ Y. Y. Zhu, N. B. Ming, W. H. Jiang, and Y. A. Shui, Appl. Phys. Lett. 53, 1381 (1988); Y. Y. Zhu and N. B. Ming, J. Appl. Phys. 72, 904 (1992); H. P. Xu, G. Z. Jiang, L. Mao, Y. Y. Zhu, M. Qi, N. B. Ming, J. H. Yin, and Y. A. Shui, ibid. 71, 2480 (1992); S. D. Cheng, Y. Y. Zhu, Y. L. Lu, and N. B. Ming, Appl. Phys. Lett. 66, 291 (1995).

${ }^{18}$ E. H. El Boudouti, B. Djafari-Rouhani, E. M. Khourdifi, and L. Dobrzynski, Phys. Rev. B 48, 10987 (1993).

${ }^{19}$ E. H. El Boudouti, B. Djafari-Rouhani, A. Akjouj, and L. Dobrzynski, Phys. Rev. B 54, 14728 (1996).

${ }^{20} \mathrm{~A}$. Bousfia (unpublished)

${ }^{21}$ E. H. El Boudouti, B. Djafari-Rouhani, and A. Nougaoui, Phys. Rev. B 51, 13801 (1995)

${ }^{22}$ D. Bria, E. H. El Boudouti, B. Djafari-Rouhani, A. Nougaoui, and V. R. Velasco, Phys. Rev. B 60, 2505 (1999).

${ }^{23}$ L. Dobrzynski, Surf. Sci. Rep. 11, 139 (1990), and references therein.

${ }^{24}$ M. Chirita, R. Sooryakumar, H. Xia, O. R. Monteiro, and I. G. Brown, Phys. Rev. B 60, R5153 (1999)

${ }^{25}$ P. Zinin, M. H. Manghnani, S. Tlechev, V. Askarpour, O. Lefeuvre, and A. Every, Phys. Rev. B 60, 2844 (1999).

${ }^{26}$ M. Moutagna, M. Ferrari, F. Rossi, F. Tonelli, and C. Tosello, Phys. Rev. B 58, R547 (1998).

${ }^{27}$ G. Tas, C. J. Morath, and H. J. Marris, Physica B 219/220, 660 (1996); W. Chen, Y. Lu, H. J. Marris, and G. Xiao, Phys. Rev. B 50, 14506 (1994).

${ }^{28}$ F. C. Jain and K. K. Bhattacharjee, Proceedings of the IEEE Ultrasonic Symposium 1990, p. 621.

${ }^{29}$ T. Gryba and J. E. Lefebvre, Proc. IEE Optoelectronics 141, 62 (1994). 